\title{
AXIOMATIC DIFFERENTIAL GEOMETRY II-2 - DIFFERENTIAL FORMS
}

\author{
HIROKAZU NISHIMURA
}

\begin{abstract}
We refurbish our axiomatics of differential geometry introduced in [5]. Then the notion of Euclideaness can naturally be formulated. The principal objective of this paper is to present an adaptation of our theory of differential forms developed in [3] to our present axiomatic framework.
\end{abstract}

\section{INTRODUCTION}

The principal objective of this paper is to replicate our treatment of differential forms in [3] in the context of our axiomatics on differential geometry in [4]. Trying to achieve this goal, we have realized that our axiomatics there is somewhat fragile. Therefore, we were forced to refurbish the axiomatics. The main improvement is that prolongations of spaces with respect to Weil algebras can directly be generalized to those with respect to finitely presented algebras. As is well known, the prolongation of a space with respect to the Weil algebra $k[X] /\left(X^{2}\right)$ (the Weil algebra corresponding to first-order infinitesimals) is its tangent bundle. Similarly, the prolongation of a space with respect to the polynomial algebra $k\left[X_{1}, \ldots, X_{n}\right]$, which is not a Weil algebra but surely a finitely presented algebra, is simply the exponentiation of the space by $\mathbb{R}^{n}$. Thus, the secondary objective in this paper is to improve our axiomatics, to which Section 2 is devoted. In particular, the theorem established in [5] that the tangent space is a module over $k$, which is external to the category $\mathcal{K}$, is enhanced to the theorem that the tangent space is a module over $\mathbb{R}$, which is an object in $\mathcal{K}$.

Section 3 is concerned with Euclidean modules. Our new axiomatics of differential geometry enables us to formulate the notion of Euclideaness properly, in which cartesian closedness and prolongations with respect to polynomial algebras will play a crucial role. In orthodox differential geometry and its extensions to infinite-dimensional differential geometry, we first study the category of linear spaces of some kind and smooth mappings, say, the category of Hilbert spaces, that of Banach spaces, that of Fréchet spaces, that of convenient vector spaces and so on. We then study the category of manifolds, which are modeled locally after such linear spaces. Our approach moves in the sheer opposite direction. We first establish the general theory of microlinear spaces. The theory of Eucliean modules (i.e., its linear part) is obtained as a special case of this general theory.

$M S C$ (2010): primary 53Z99, $18 \mathrm{~F} 99$.

Keywords: axiomatic differential geometry, differential form, Weil algebra, finitely presented algebra, Euclidean module. 
Sections 4 and 5 are merely an adaptation of our treatment of differential forms in [5] to our present axiomatic framework. Section 4 is devoted to a unique characterization of differential forms, which could be called the fundamental theorem on differential forms. The characterization and existence of exterior differentiation, which will be discussed in Section 5, is an easy consequence of this fundamental theorem.

\section{Refurbishing our AXIOMATics}

\subsection{The refurbishment}

Let $k$ be a commutative ring. We denote by $\mathbb{T}_{k}$ the algebraic theory of $k$-algebras in the sense of Lawvere. We denote by $\mathbf{F P} \mathbb{T}_{k}$ the category of finitely presented $k$-algebras. It is well known that Weil algebras over $k$ are finitely presented $k$ algebras. We denote by Weil $_{k}$ the category of Weil $k$-algebras, which is well known to be left exact. In particular, its terminal object is $k$ itself. A finitely presented $k$-algebra $A$ is called pointed if it has a unique maximal ideal $\mathfrak{m}$ such that the composition of the canonical morphism

$$
k \rightarrow A
$$

and the canonical projection

$$
A \rightarrow A / \mathfrak{m}
$$

is an isomorphism. We denote by $\mathbf{P F P} \mathbb{T}_{k}$ the category of pointed finitely presented $k$-algebras. Not only Weil $k$-algebras but also polynomial $k$-algebras $k\left[X_{1}, \ldots, X_{n}\right]$ lie in $\mathbf{P F P} \mathbb{T}_{k}$. Given a left exact category $\mathcal{K}$ and a $k$-algebra object $\mathbb{R}$ in $\mathcal{K}$, there is a canonical functor $\mathbb{R} \underline{\otimes}$. (denoted by $\mathbb{R} \otimes \cdot$ in [1]) from the category $\mathbf{W e i l}_{k}$ to the category of $k$-algebra objects and their homomorphisms in $\mathcal{K}$.

Definition 2.1 (DG-category). The present refinement of our original axiomatics in [4] is that we allow not only Weil prolongations but also finitely presented prolongations. Therefore, given a finitely presented $k$-algebra $A$, we are endowed with a left exact functor $\mathbf{T}^{A}: \mathcal{K} \rightarrow \mathcal{K}$ preserving cartesian closed structures in the sense that we have

$$
\mathbf{T}^{A}\left(X^{Y}\right)=\left(\mathbf{T}^{A} X\right)^{Y}
$$

for any objects $X$ and $Y$ in $\mathcal{K}$. For any freely generated $k$-algebra $A=k\left[X_{1}, \ldots, X_{n}\right]$ over $n$ generaters $X_{1}, \ldots, X_{n}, \mathbf{T}^{A}=\mathbf{T}^{k\left[X_{1}, \ldots, X_{n}\right]}$ is required to be simply the exponentiation by $\mathbb{R}^{n}$, so that we have

$$
\mathbf{T}^{k\left[X_{1}, \ldots, X_{n}\right]} X=X^{\mathbb{R}^{n}}
$$

for any object $X$ in $\mathcal{K}$. In particular, when $n=0$, we have

$$
\mathbf{T}^{k} X=X \text {. }
$$

Given a finitely presented $k$-algebra $A$, it is required that

$$
\mathbf{T}^{A} \mathbb{R}=\underline{R} \otimes A .
$$

Given two finitely presented $k$-algebra $A$ and $B$, it is required that

$$
\mathbf{T}^{B} \circ \mathbf{T}^{A}=\mathbf{T}^{A \otimes_{k} B} .
$$


Given a morphism $\varphi: A \rightarrow B$ in $\mathbf{P F P} \mathbb{T}_{k}$, we have a natural transformation

$$
\alpha_{\varphi}: \mathbf{T}^{A} \Rightarrow \mathbf{T}^{B}
$$

which respects cartesian closed structures, so that we have

$$
\alpha_{\varphi}\left(X^{Y}\right)=\left(\alpha_{\varphi}(X)\right)^{Y}
$$

for any objects $X$ and $Y$ in $\mathcal{K}$. It is also required to satisfy

$$
\begin{aligned}
& \alpha_{\varphi}\left(\mathbf{T}^{C} X\right)=\mathbf{T}^{C}\left(\alpha_{\varphi}(X)\right): \mathbf{T}^{A} \mathbf{T}^{C} X=\mathbf{T}^{C \otimes_{k} A} X=\mathbf{T}^{A \otimes_{k} C} X \\
= & \mathbf{T}^{C} \mathbf{T}^{A} X \rightarrow \mathbf{T}^{C} \mathbf{T}^{B} X=\mathbf{T}^{B \otimes_{k} C} X=\mathbf{T}^{C \otimes_{k} B} X=\mathbf{T}^{B} \mathbf{T}^{C} X
\end{aligned}
$$

for any object $C$ in the category $\mathbf{P F P} \mathbb{T}_{k}$. Given two morphisms $\varphi: A \rightarrow B$ and $\psi: B \rightarrow C$ in $\mathbf{P F P} \mathbb{T}_{k}$, it is required that

$$
\alpha_{\psi} \circ \alpha_{\varphi}=\alpha_{\psi \circ \varphi}
$$

Given any identity morphism $\operatorname{id}_{A}: A \rightarrow A$ in $\mathbf{P F P} \mathbb{T}_{k}$, it is required that

$$
\alpha_{\mathrm{id}_{A}}=\operatorname{id}_{\mathbf{T}^{A}} .
$$

Given a morphism $\varphi: A \rightarrow B$ in $\mathbf{P F P} \mathbb{T}_{k}$, it is required that

$$
\alpha_{\varphi}(\mathbb{R})=\mathbb{R} \underline{\otimes} \varphi .
$$

Thus our new definition of a DG-category is a quadruple

$$
(\mathcal{K}, \mathbb{R}, \mathbf{T}, \alpha),
$$

where

(1) $\mathcal{K}$ is a category which is left exact and cartesian closed,

(2) $\mathbb{R}$ is a commutative $k$-algebra object in $\mathcal{K}$,

(3) given an object $A$ in $\mathbf{P F P} \mathbb{T}_{k}, \mathbf{T}^{A}: \mathcal{K} \rightarrow \mathcal{K}$ is a left-exact and cartesianclosed-structure-preserving functor,

(4) given a morphism $\varphi: A \rightarrow B$ in $\mathbf{P F P} \mathbb{T}_{k}, \alpha_{\varphi}: \mathbf{T}^{A} \Rightarrow \mathbf{T}^{B}$ is a natural transformation,

(5) the quadruple $(\mathcal{K}, \mathbb{R}, \mathbf{T}, \alpha)$ is required to satisfy (2.2)-(2.9) as axioms.

Remark 2.2. As in [4], we have a bifunctor

$$
\otimes: \mathcal{K} \times \mathbf{P F P} \mathbb{T}_{k} \rightarrow \mathcal{K}
$$

with

$$
X \otimes A=\mathbf{T}^{A} X
$$

for any object $X$ in $\mathcal{K}$ and any object $A$ in $\mathbf{P F P} \mathbb{T}_{k}$, and

$$
f \otimes \varphi=\alpha_{\varphi}(Y) \circ \mathbf{T}^{A} f=\mathbf{T}^{B} f \circ \alpha_{\varphi}(X)
$$

for any morphism

$$
f: X \rightarrow Y
$$

in $\mathcal{K}$ and any morphism

$$
\varphi: A \rightarrow B
$$

in $\mathbf{P F P} \mathbb{T}_{k}$. 
Remark 2.3. Given an object $A$ in $\mathbf{P F P} \mathbb{T}_{k}$ and an object $X$ in $\mathcal{K}$, we write

$$
\tau_{A}(X): \mathbf{T}^{A} X \rightarrow X
$$

and

$$
\iota_{A}(X): X \rightarrow \mathbf{T}^{A} X
$$

for

and

$$
\alpha_{A \rightarrow k}(X): \mathbf{T}^{A} X \rightarrow \mathbf{T}^{k} X=X
$$

$$
\alpha_{k \rightarrow A}(X): X=\mathbf{T}^{k} X \rightarrow \mathbf{T}^{A} X
$$

respectively, where $A \rightarrow k$ and $k \rightarrow A$ are the canonical morphisms in $\mathbf{P F P} \mathbb{T}_{k}$.

It is easy to see that

Proposition 2.4. Let $(\mathcal{K}, \mathbb{R}, \mathbf{T}, \alpha)$ be a $D G$-category with the category $\mathcal{K}$ being locally cartesian closed and $M$ an object in $\mathcal{K}$. Then $\left(\mathcal{K} / M, \mathbb{R}_{M}, \mathbf{T}_{M}, \alpha^{M}\right)$ is a DG-category but for conditions (2.1) and (2.5), where

(1) $\mathcal{K} / M$ is the slice category,

(2) $\mathbb{R}_{M}$ is the canonical projection

$$
\mathbb{R} \times M \rightarrow M,
$$

(3) given an object

$$
\pi: E \rightarrow M
$$

in $\mathcal{K}$ and an object $A$ in $\mathbf{P F P} \mathbb{T}_{k}, \mathbf{T}_{M}^{A}(\pi)$ is defined to be

$$
\overline{\mathbf{T}}_{M}^{A}(\pi) \rightarrow M
$$

where $\overline{\mathbf{T}}_{M}^{A}(\pi)$ is obtained as the equalizer of

$$
\mathbf{T}^{A} \pi: \mathbf{T}^{A} E \rightarrow \mathbf{T}^{A} M
$$

and

$$
\mathbf{T}^{A} E \stackrel{\tau_{A}(E)}{E} \underset{\rightarrow}{\rightarrow} M \stackrel{\iota_{A}(M)}{\longrightarrow} \mathbf{T}^{A} M
$$

and (2.10) is

$$
\overline{\mathbf{T}}_{M}^{A}(\pi) \rightarrow \mathbf{T}^{A} E \underline{\tau_{A}(E)} E \stackrel{\pi}{\rightarrow} M,
$$

(4) let $\varphi: A \rightarrow B$ be a morphism in $\mathbf{P F P} \mathbb{T}_{k}$. Since the diagrams

$$
\begin{array}{ccc}
\mathbf{T}^{A} E & \alpha_{\varphi}(E) & \mathbf{T}^{B} E \\
\mathbf{T}^{A} \pi \downarrow & & \downarrow \mathbf{T}^{B} \pi \\
\mathbf{T}^{A} M & \overrightarrow{\alpha_{\varphi}(M)} & \mathbf{T}^{B} M \\
\mathbf{T}^{A} E & \alpha_{\varphi}(E) & \mathbf{T}^{B} E \\
\tau_{A}(E) \searrow & & \swarrow \tau_{B}(E) \\
& E & \\
& \pi \downarrow & \\
\iota_{A}(M) \swarrow & \stackrel{ }{\mathbf{T}_{\varphi}(M)} & \searrow \iota_{B}(M) \\
\mathbf{T}^{A} M & \mathbf{T}^{B} M
\end{array}
$$


commute, there is a unique morphism

$$
\alpha_{\varphi}^{M}(\pi): \overline{\mathbf{T}}_{M}^{A}(\pi) \rightarrow \overline{\mathbf{T}}_{M}^{B}(\pi)
$$

in $\mathcal{K}$ such that the diagram

$$
\begin{array}{ccc}
\overline{\mathbf{T}}_{M}^{A}(\pi) & \stackrel{\alpha_{\varphi}^{M}(\pi)}{\longrightarrow} & \overline{\mathbf{T}}_{M}^{B}(\pi) \\
\downarrow & & \downarrow \\
\mathbf{T}^{A} E & \overrightarrow{\alpha_{\varphi}(E)} & \mathbf{T}^{B} E
\end{array}
$$

commutes.

Definition 2.5 (Local DG-category). A DG-category $(\mathcal{K}, \mathbb{R}, \mathbf{T}, \alpha)$ is called a local $D G$-category if $\mathcal{K}$ is locally cartesian closed and $\left(\mathcal{K} / M, \mathbb{R}_{M}, \mathbf{T}_{M}, \alpha^{M}\right)$ is a DGcategory for any object $M$ in $\mathcal{K}$.

Remark 2.6. The notion of microlinearity and that of Weil exponentiability remain the same as those in [4].

In the following, we will consider an arbitrarily chosen local DG-category $(\mathcal{K}, \mathbb{R}, \mathbf{T}, \alpha)$ with $M$ being a microlinear and Weil exponentiable object in $\mathcal{K}$.

\subsection{The duality}

We have already explained the duality between the category of Weil algebras in the real world and the category of infinitesimal objects in the imaginary world. Namely, we have a contravariant functor $\mathcal{D}$ from the category of Weil algebras to the category of infinitesimal objects and a contravariant functor $\mathcal{W}$ from the category of infinitesimal objects to the category of Weil algebras, both of which constitute a dual equivalence between the two categories. By way of example, $\mathcal{D}_{k[X] /\left(X^{2}\right)}$ is intended for

$$
D=\left\{x \in \mathbb{R} \mid x^{2}=0\right\}
$$

while $\mathcal{D}_{k[X, Y] /\left(X^{2}, Y^{2}, X Y\right)}$ is intended for

$$
D(2)=\{(x, y) \in D \times D \mid x y=0\} .
$$

Therefore, we have

$$
\begin{aligned}
& \mathcal{W}_{D}=k[X] /\left(X^{2}\right) \\
& \mathcal{W}_{D(2)}=k[X, Y] /\left(X^{2}, Y^{2}, X Y\right)
\end{aligned}
$$

Similarly

$$
\mathcal{W}_{d \in D \mapsto(d, 0) \in D(2)}
$$

stands for the homomorphism of $k$-algebras from $k[X, Y] /\left(X^{2}, Y^{2}, X Y\right)$ to $k[X] /$ $\left(X^{2}\right)$ assigning the equivalence class of $X$ in $k[X] /\left(X^{2}\right)$ to the equivalence class of $X$ in $[X, Y] /\left(X^{2}, Y^{2}, X Y\right)$ and assigning the equivalence class of 0 in $k[X] /\left(X^{2}\right)$ to the equivalence class of $Y$ in $[X, Y] /\left(X^{2}, Y^{2}, X Y\right)$.

We can extend contravariant functors $\mathcal{D}$ and $\mathcal{W}$ so as to yield a dual equivalence between the category $\mathbf{P F P} \mathbb{T}_{k}$ and the category of (real or imaginary) carved spaces 
standing for genuinely formal $\mathrm{Spec}_{\mathbb{R}}$. By way of example, we have

$$
\begin{aligned}
& \mathcal{D}_{k\left[Z_{1}, Z_{2}\right]}=\mathbb{R}^{2}, \\
& \mathcal{D}_{k\left[Z_{1}, Z_{2}, X, Y\right] /\left(X^{2}, Y^{2}, X Y\right)}=\mathbb{R}^{2} \times D(2),
\end{aligned}
$$

while

$$
\mathcal{W}_{d \in D \mapsto d \in \mathbb{R}}
$$

stands for the canonical projection

$$
k[X] \rightarrow k[X] /\left(X^{2}\right)
$$

and

$$
\mathcal{W}_{\left(r_{1}, r_{2}, d_{1}, d_{2}\right) \in \mathbb{R}^{2} \times D(2) \mapsto r_{1} d_{1}+r_{2} d_{2} \in D}
$$

stands for the homomorphism of $k$-algebras from $k[X] /\left(X^{2}\right)$ to $k\left[Z_{1}, Z_{2}, X, Y\right] /$ $\left(X^{2}, Y^{2}, X Y\right)$ assigning the equivalence class of $Z_{1} X+Z_{2} Y$ in $k\left[Z_{1}, Z_{2}, X, Y\right] /$ $\left(X^{2}, Y^{2}, X Y\right)$ to the equivalence class of $X$ in $k[X] /\left(X^{2}\right)$.

\subsection{The tangent space}

Definition 2.7 (Scalar Multiplication). The exponential transpose of the scalar multiplication

$$
\mathbb{R} \times\left(M \otimes \mathcal{W}_{D}\right) \rightarrow M \otimes \mathcal{W}_{D}
$$

is

$$
\begin{aligned}
& \operatorname{id}_{M} \otimes \mathcal{W}_{(r, d) \in \mathbb{R} \times D \mapsto r d \in D}: M \otimes \mathcal{W}_{D} \rightarrow M \otimes \mathcal{W}_{\mathbb{R} \times D}=M \otimes\left(\mathcal{W}_{D} \otimes_{k} \mathcal{W}_{\mathbb{R}}\right) \\
= & \left(M \otimes \mathcal{W}_{D}\right) \otimes \mathcal{W}_{\mathbb{R}}=\left(M \otimes \mathcal{W}_{D}\right)^{\mathbb{R}} .
\end{aligned}
$$

Now we strengthen one of the main results of [5] into

Theorem 2.8. The canonical projection

$$
\tau_{\mathcal{W}_{D}}(M): M \otimes \mathcal{W}_{D} \rightarrow M
$$

is an $\mathbb{R}_{M}$-module in the slice category $\mathcal{K} / M$, where $\mathbb{R}_{M}$ is the canonical projection

$$
\mathbb{R} \times M \rightarrow M .
$$

Proof. Here we deal only with the statement that the scalar multiplication distributes over the addition, for which we have to verify that the diagram

$$
\begin{array}{cccc}
\mathbb{R} \times\left(M \otimes \mathcal{W}_{D(2)}\right) & \rightarrow & \mathbb{R} \times\left(M \otimes \mathcal{W}_{D}\right) \\
\downarrow & & \downarrow \\
M \otimes \mathcal{W}_{D(2)} & \rightarrow & M \otimes \mathcal{W}_{D}
\end{array}
$$

commutes, where the horizontal arrows stand for addition, while the vertical arrows correspond to scalar multiplication. This follows easily from the commutativity of the diagram

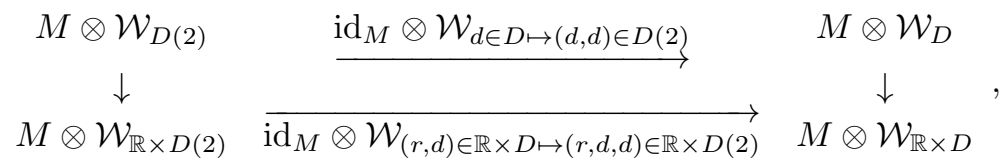

where the left vertical arrow is

$$
\operatorname{id}_{M} \otimes \mathcal{W}_{\left(r, d_{1}, d_{2}\right) \in \mathbb{R} \times D \mapsto\left(r d_{1}, r d_{2}\right) \in D},
$$


while the right vertical arrow is

$$
\operatorname{id}_{M} \otimes \mathcal{W}_{(r, d) \in \mathbb{R} \times D \mapsto r d \in D} .
$$

\section{EuClidean Modules}

An $\mathbb{R}$-module in $\mathcal{K}$ is an object $\mathbb{E}$ in $\mathcal{K}$ endowed with a morphism

$$
+_{\mathbb{E}}: \mathbb{E} \times \mathbb{E} \rightarrow \mathbb{E}
$$

intended for addition, and a morphism

$$
\cdot \mathbb{R}, \mathbb{E}: \mathbb{R} \times \mathbb{E} \rightarrow \mathbb{E}
$$

intended for scalar multiplication, which are surely subject to the usual axioms of an $\mathbb{R}$-module depicted diagrammatically. Equivalently, an $\mathbb{R}$-module structure on an object $\mathbb{E}$ in $\mathcal{K}$ can be given by a single morphism

$$
\varphi: \mathbb{R} \times \mathbb{E} \times \mathbb{E} \rightarrow \mathbb{E}
$$

intended for the morphism

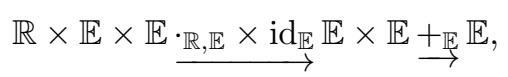

which is surely subject to some axioms depicted diagrammatically.

Definition 3.1 (Euclidean $\mathbb{R}$-module). An $\mathbb{R}$-module $\mathbb{E}$ is called Euclidean provided that the composition of the exponential transpose

$$
\mathbb{E} \times \mathbb{E} \rightarrow \mathbb{E}^{\mathbb{R}}
$$

of

$$
\mathbb{R} \times \mathbb{E} \times \mathbb{E}=\mathbb{E} \times \mathbb{R} \times \underset{\mathbb{E} i \mathrm{id}_{\mathbb{E}} \times \mathbb{R , \mathbb { E }}}{\longrightarrow} \mathbb{E} \times \stackrel{\mathbb{E}}{\longrightarrow} \mathbb{E}
$$

and

$$
\alpha_{\mathcal{W}_{d \in D \mapsto d \in \mathbb{R}}}(\mathbb{E}): \mathbb{E}^{\mathbb{R}}=\mathbb{E} \otimes \mathcal{W}_{\mathbb{R}} \rightarrow \mathbb{E} \otimes \mathcal{W}_{D}
$$

in succession is an isomorphism.

It should be obvious that

Lemma 3.2. The $\mathbb{R}$-module structure of $\mathbb{E}$ naturally gives rise to that of $\mathbb{E}^{X}$ for any object $X$ in $\mathcal{K}$ in the sense that the exponential transpose

$$
\widetilde{\varphi}: \mathbb{E} \times \mathbb{E} \rightarrow \mathbb{E}^{\mathbb{R}}
$$

of the $\mathbb{R}$-module structure

$$
\varphi: \mathbb{R} \times \mathbb{E} \times \mathbb{E} \rightarrow \mathbb{E}
$$

on $\mathbb{E}$ induces a mapping

$$
(\widetilde{\varphi})^{X}: \mathbb{E}^{X} \times \mathbb{E}^{X}=(\mathbb{E} \times \mathbb{E})^{X} \rightarrow\left(\mathbb{E}^{\mathbb{R}}\right)^{X}=\left(\mathbb{E}^{X}\right)^{\mathbb{R}}
$$

which is the exponential transpose of the derived $\mathbb{R}$-module structure

$$
\mathbb{R} \times \mathbb{E}^{X} \times \mathbb{E}^{X} \rightarrow \mathbb{E}^{X}
$$

on $\mathbb{E}^{X}$. 
Proposition 3.3. If $\mathbb{E}$ is a Euclidean $\mathbb{R}$-module, then so is $\mathbb{E}^{X}$ for any object $X$ in $\mathcal{K}$.

Proof. We use the same notation as in Lemma 3.2. We have

$$
\begin{aligned}
& \alpha_{\mathcal{W}_{d \in D \mapsto d \in \mathbb{R}}}\left(\mathbb{E}^{X}\right)=\left(\alpha_{\mathcal{W}_{d \in D \mapsto d \in \mathbb{R}}}(\mathbb{E})\right)^{X}:\left(\mathbb{E}^{X}\right)^{\mathbb{R}}=\left(\mathbb{E}^{\mathbb{R}}\right)^{X}=\left(\mathbb{E} \otimes \mathcal{W}_{\mathbb{R}}\right)^{X} \\
\rightarrow & \left(\mathbb{E} \otimes \mathcal{W}_{D}\right)^{X}=\mathbb{E}^{X} \otimes \mathcal{W}_{D} .
\end{aligned}
$$

Since

$$
\left(\alpha_{\mathcal{W}_{d \in D \mapsto d \in \mathbb{R}}}(\mathbb{E})\right)^{X} \circ(\widetilde{\varphi})^{X}=\left(\alpha_{\mathcal{W}_{d \in D \mapsto d \in \mathbb{R}}}(\mathbb{E}) \circ \widetilde{\varphi}\right)^{X},
$$

we are sure that $\mathbb{E}^{X}$ is a Euclidean $\mathbb{R}$-module.

It should be evident that

Lemma 3.4. The $\mathbb{R}$-module structure of $\mathbb{E}$ naturally gives rise to that of $\mathbb{E} \otimes W$ for any Weil algebra $W$ in the sense that the exponential transpose

$$
\widetilde{\varphi}: \mathbb{E} \times \mathbb{E} \rightarrow \mathbb{E}^{\mathbb{R}}
$$

of the $\mathbb{R}$-module structure

$$
\varphi: \mathbb{R} \times \mathbb{E} \times \mathbb{E} \rightarrow \mathbb{E}
$$

on $\mathbb{E}$ induces a mapping

$$
\widetilde{\varphi} \otimes \operatorname{id}_{W}:(\mathbb{E} \otimes W) \times(\mathbb{E} \otimes W)=(\mathbb{E} \times \mathbb{E}) \otimes W \rightarrow \mathbb{E}^{\mathbb{R}} \otimes W=(\mathbb{E} \otimes W)^{\mathbb{R}},
$$

which is the exponential transpose of the derived $\mathbb{R}$-module structure

$$
\mathbb{R} \times(\mathbb{E} \otimes W) \times(\mathbb{E} \otimes W) \rightarrow \mathbb{E} \otimes W
$$

on $\mathbb{E} \otimes W$.

Proposition 3.5. If $\mathbb{E}$ is a Euclidean $\mathbb{R}$-module, then so is $\mathbb{E} \otimes W$ for any Weil algebra $W$.

Proof. We use the same notation as in Lemma 3.4. We have

$$
\begin{aligned}
& \alpha_{\mathcal{W}_{d \in D \mapsto d \in \mathbb{R}}}(\mathbb{E} \otimes W)=\alpha_{\mathcal{W}_{d \in D \mapsto d \in \mathbb{R}}}(\mathbb{E}) \otimes \mathrm{id}_{W}:(\mathbb{E} \otimes W)^{\mathbb{R}}=(\mathbb{E} \otimes W) \otimes \mathcal{W}_{\mathbb{R}} \\
= & \left(\mathbb{E} \otimes \mathcal{W}_{\mathbb{R}}\right) \otimes W \rightarrow\left(\mathbb{E} \otimes \mathcal{W}_{D}\right) \otimes W=(\mathbb{E} \otimes W) \otimes \mathcal{W}_{D} .
\end{aligned}
$$

Since

$$
\left(\alpha_{\mathcal{W}_{d \in D \mapsto d \in \mathbb{R}}}(\mathbb{E}) \otimes \mathrm{id}_{W}\right) \circ\left(\widetilde{\varphi} \otimes \mathrm{id}_{W}\right)=\left(\alpha_{\mathcal{W}_{d \in D \mapsto d \in \mathbb{R}}}(\mathbb{E}) \circ \widetilde{\varphi}\right) \otimes \mathrm{id}_{W},
$$

we are sure that $\mathbb{E} \otimes W$ is a Euclidean $\mathbb{R}$-module.

Remark 3.6. If $\mathbb{E}$ is an $\mathbb{R}$-module, then the first projection

$$
\pi_{1}: \mathbb{E} \times \mathbb{E} \rightarrow \mathbb{E}
$$

is naturally an $\mathbb{R}$-module in the slice category $\mathcal{K} / \mathbb{E}$. 
Proposition 3.7. If $\mathbb{E}$ is a Euclidean $\mathbb{R}$-module, then the identification of $\mathbb{E} \otimes$ $\mathcal{W}_{D}$ and $\mathbb{E} \times \mathbb{E}$ in Definition 3.1 together with the commutative diagram

$$
\begin{array}{ccc}
\mathbb{E} \times \mathbb{E} & = & \mathbb{E} \otimes \mathcal{W}_{D} \\
\pi_{1} \searrow & & \swarrow \tau_{\mathcal{W}_{D}}(\mathbb{E})
\end{array}
$$

allows us to identify the $\mathbb{R}_{M}$-module structure in Theorem 2.8 and that in Remark 3.6.

Proof. (1) First we deal with addition. We have

$$
\begin{aligned}
& \mathbb{E} \otimes W_{D(2)}=\left(\mathbb{E} \otimes \mathcal{W}_{D}\right) \times_{M}\left(\mathbb{E} \otimes \mathcal{W}_{D}\right) \\
= & (\underset{1}{\mathbb{E}} \times \underset{2}{\mathbb{E}}) \times_{M}(\underset{3}{\mathbb{E}} \times \underset{4}{\mathbb{E}}) \\
= & \underset{1,3}{\mathbb{E}} \times \underset{2}{\mathbb{E}} \times \underset{4}{\mathbb{E}}
\end{aligned}
$$

where the numbers under $\mathbb{E}$ are given simply for the reader to relate each occurrence of $\mathbb{E}$ on the last line to the appropriate occurrence of $\mathbb{E}$ on the previous line. This isomorphism can be realized by the composition of the exponential transpose

$$
\mathbb{E} \times \mathbb{E} \times \mathbb{E} \rightarrow \mathbb{E}^{\mathbb{R} \times \mathbb{R}}
$$

of

$$
\begin{aligned}
& \underset{1}{\mathbb{R}} \times \underset{2}{\mathbb{R}} \times \underset{3}{\mathbb{E}} \times \underset{4}{\mathbb{E}} \times \underset{5}{\mathbb{E}}=\underset{3}{\mathbb{E}} \times \underset{1}{\mathbb{R}} \times \underset{4}{\mathbb{E}} \times \underset{2}{\mathbb{R}} \times \underset{5}{\mathbb{E}} \underset{\mathbb{E}}{\operatorname{id}} \times \cdot \mathbb{R}, \mathbb{E} \times \cdot \underset{\mathbb{R}, \mathbb{E}}{\longrightarrow} \mathbb{E} \times \mathbb{E} \times \mathbb{E} \\
& \stackrel{\mathrm{id}_{\mathbb{E}} \times+_{\mathbb{E}}}{\longrightarrow} \mathbb{E} \times \underset{+}{\longrightarrow} \mathbb{E}
\end{aligned}
$$

and

$$
\alpha_{\mathcal{W}_{\left(d_{1}, d_{2}\right) \in D(2) \mapsto\left(d_{1}, d_{2}\right) \in \mathbb{R} \times \mathbb{R}}}(\mathbb{E}): \mathbb{E}^{\mathbb{R} \times \mathbb{R}}=\mathbb{E} \otimes \mathcal{W}_{\mathbb{R} \times \mathbb{R}} \rightarrow \mathbb{E} \otimes \mathcal{W}_{D(2)}
$$

in succession, where the numbers under $\mathbb{R}$ and $\mathbb{E}$ are intended for the reader to easily relate their occurrences on the first line to those on the second line. Therefore, the commutativity of the diagrams

$$
\begin{aligned}
& \mathbb{E} \times \mathbb{E} \times \mathbb{E} \quad \rightarrow \quad \mathbb{E}^{\mathbb{R} \times \mathbb{R}}=\mathbb{E} \otimes \mathcal{W}_{\mathbb{R} \times \mathbb{R}} \\
& \operatorname{id}_{\mathbb{E}} \times+_{\mathbb{E}} \downarrow \quad \downarrow \operatorname{id}_{\mathbb{E}} \otimes \mathcal{W}_{r \in \mathbb{R} \mapsto(r, r) \in \mathbb{R} \times \mathbb{R}} \\
& \mathbb{E} \times \mathbb{E} \quad \rightarrow \quad \mathbb{E}^{\mathbb{R}}=\mathbb{E} \otimes \mathcal{W}_{\mathbb{R}} \\
& \mathbb{E}^{\mathbb{R} \times \mathbb{R}}=\mathbb{E} \otimes \mathcal{W}_{\mathbb{R} \times \mathbb{R}} \quad \rightarrow \quad \mathbb{E} \otimes \mathcal{W}_{D(2)} \\
& \operatorname{id}_{\mathbb{E}} \otimes \mathcal{W}_{r \in \mathbb{R} \mapsto(r, r) \in \mathbb{R} \times \mathbb{R}} \downarrow \quad \downarrow \quad \operatorname{id}_{\mathbb{E}} \otimes \mathcal{W}_{d \in D \mapsto(d, d) \in D(2)} \quad, \\
& \mathbb{E}^{\mathbb{R}}=\mathbb{E} \otimes \mathcal{W}_{\mathbb{R}} \quad \rightarrow \quad \mathbb{E} \otimes \mathcal{W}_{D}
\end{aligned}
$$

with the morphism

$$
\mathbb{E} \times \mathbb{E} \times \mathbb{E} \rightarrow \mathbb{E}^{\mathbb{R} \times \mathbb{R}}
$$

being that in (3.2), the morphism

$$
\mathbb{E} \times \mathbb{E} \rightarrow \mathbb{E}^{\mathbb{R}}
$$

being that in (3.1), the moriphism

$$
\mathbb{E}^{\mathbb{R} \times \mathbb{R}}=\mathbb{E} \otimes \mathcal{W}_{\mathbb{R} \times \mathbb{R}} \rightarrow \mathbb{E} \otimes \mathcal{W}_{D(2)}
$$


being the morphism

$$
\operatorname{id}_{\mathbb{E}} \otimes \mathcal{W}_{\left(d_{1}, d_{2}\right) \in D(2) \mapsto\left(d_{1}, d_{2}\right) \in \mathbb{R} \times \mathbb{R}}
$$

and the morphism

$$
\mathbb{E}^{\mathbb{R}}=\mathbb{E} \otimes \mathcal{W}_{\mathbb{R}} \rightarrow \mathbb{E} \otimes \mathcal{W}_{D}
$$

being the morphism

$$
\operatorname{id}_{\mathbb{E}} \otimes \mathcal{W}_{d \in D \mapsto d \in \mathbb{R}}
$$

implies the commutativity of the diagram

$$
\begin{array}{cccc}
\mathbb{E} \times \mathbb{E} \times \mathbb{E} & = & \mathbb{E} \otimes \mathcal{W}_{D(2)} \\
\operatorname{id}_{\mathbb{E}} \times+_{\mathbb{E}} \downarrow & & \downarrow & \operatorname{id}_{\mathbb{E}} \otimes \mathcal{W}_{d \in D \mapsto(d, d) \in D(2)} \\
\mathbb{E} \times \mathbb{E} & = & \mathbb{E} \otimes \mathcal{W}_{D}
\end{array} .
$$

This is nothing else but the gist of the desired statement.

(2) Now we deal with scalar multiplication. The commutativity of the diagrams

$$
\begin{array}{cccc}
\mathbb{E} \times \mathbb{E} & \rightarrow & \mathbb{E}^{\mathbb{R}}=\mathbb{E} \otimes \mathcal{W}_{\mathbb{R}} \\
\downarrow & & \downarrow \operatorname{id}_{\mathbb{E}} \otimes \mathcal{W}_{\left(r_{1}, r_{2}\right) \in \mathbb{R} \times \mathbb{R} \mapsto r_{1} r_{2} \in \mathbb{R}} \\
(\mathbb{E} \times \mathbb{E})^{\mathbb{R}} & \rightarrow & \mathbb{E}^{\mathbb{R} \times \mathbb{R}}=\mathbb{E} \otimes \mathcal{W}_{\mathbb{R} \times \mathbb{R}} \\
\mathbb{E}^{\mathbb{R}}=\mathbb{E} \otimes \mathcal{W}_{\mathbb{R}} & \rightarrow & \mathbb{E} \otimes \mathcal{W}_{D} \\
\operatorname{id}_{\mathbb{E}} \otimes \mathcal{W}_{\left(r_{1}, r_{2}\right) \in \mathbb{R} \times \mathbb{R} \mapsto r_{1} r_{2} \in \mathbb{R}} \downarrow & & \downarrow \operatorname{id}_{\mathbb{E}} \otimes \mathcal{W}_{(d, r) \in D \times \mathbb{R} \mapsto d r \in D} \\
\mathbb{E}^{\mathbb{R} \times \mathbb{R}=}=\mathbb{E} \otimes \mathcal{W}_{\mathbb{R} \times \mathbb{R}} & \rightarrow & \mathbb{E} \otimes \mathcal{W}_{D \times \mathbb{R}}=\left(\mathbb{E} \otimes \mathcal{W}_{D}\right)^{\mathbb{R}}
\end{array}
$$

with the left vertical arrow in the first diagram

$$
\mathbb{E} \times \mathbb{E} \rightarrow(\mathbb{E} \times \mathbb{E})^{\mathbb{R}}
$$

being the exponential transpose of

$$
\mathbb{R} \times \mathbb{E} \times \mathbb{E}=\mathbb{E} \times \mathbb{R} \times \mathbb{E} \underset{\mathbb{i d}}{\stackrel{\mathbb{E}}{\mathrm{in}_{\mathbb{R}, \mathbb{E}}}} \mathbb{E} \times \mathbb{E}
$$

the upper horizontal arrow

$$
\mathbb{E} \times \mathbb{E} \rightarrow \mathbb{E}^{\mathbb{R}}
$$

in the first diagram being that in (3.1), the lower horizontal arrow

$$
(\mathbb{E} \times \mathbb{E})^{\mathbb{R}} \rightarrow \mathbb{E}^{\mathbb{R} \times \mathbb{R}}=\mathbb{E} \otimes \mathcal{W}_{\mathbb{R} \times \mathbb{R}}
$$

in the first diagram being that in (3.1) exponentiated by $\mathbb{R}$, the upper horizontal arrow

$$
\mathbb{E}^{\mathbb{R}}=\mathbb{E} \otimes \mathcal{W}_{\mathbb{R}} \rightarrow \mathbb{E} \otimes \mathcal{W}_{D}
$$

in the second diagram being

$$
\operatorname{id}_{\mathbb{E}} \otimes \mathcal{W}_{d \in D \mapsto d \in \mathbb{R}}
$$

and the lower horizontal arrow

$$
\mathbb{E}^{\mathbb{R} \times \mathbb{R}}=\mathbb{E} \otimes \mathcal{W}_{\mathbb{R} \times \mathbb{R}} \rightarrow \mathbb{E} \otimes \mathcal{W}_{D \times \mathbb{R}}=\left(\mathbb{E} \otimes \mathcal{W}_{D}\right)^{\mathbb{R}}
$$

in the second diagram being

$$
\operatorname{id}_{\mathbb{E}} \otimes \mathcal{W}_{(d, r) \in D \times \mathbb{R} \mapsto(d, r) \in \mathbb{R} \times \mathbb{R}}
$$


implies the commutativity of the diagram

$$
\begin{array}{ccc}
\mathbb{E} \times \mathbb{E} & = & \mathbb{E} \otimes \mathcal{W}_{D} \\
\downarrow & & \downarrow \\
(\mathbb{E} \times \mathbb{E})^{\mathbb{R}} & = & \left(\mathbb{E} \otimes \mathcal{W}_{D}\right)^{\mathbb{R}}
\end{array}
$$

which is the exponential transpose of the commutative diagram

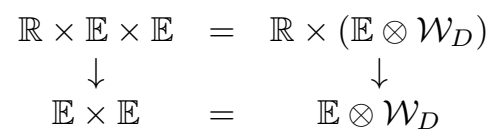

with the left vertical arrow being that in (3.3) and the right vertical arrow being the scalar multiplication in Definition 2.7. This is nothing else but the gist of the desired statement.

It should be apparent that

Lemma 3.8. The diagram

$$
\mathcal{W}_{D(2)} \stackrel{\mathcal{W}_{\left(d_{1}, d_{2}\right) \in D^{2} \mapsto\left(d_{1}, d_{1} d_{2}\right) \in D(2)}}{\mathcal{W}_{D^{2}}} \underset{\mathcal{W}_{d \in D \mapsto(0, d) \in D^{2}}}{\stackrel{\mathcal{W}_{d \in D \mapsto(0,0) \in D^{2}}}{\longrightarrow}} \mathcal{W}_{D}
$$

is a limit diagram in the category $\mathbf{W e i l}_{k}$.

Theorem 3.9. The $\mathbb{R}_{M}$-module

$$
\tau_{\mathcal{W}_{D}}(M): M \otimes \mathcal{W}_{D} \rightarrow M
$$

is Euclidean with respect to the DG-category $\left(\mathcal{K} / M, \mathbb{R}_{M}, \mathbf{T}_{M}, \alpha^{M}\right)$.

Proof. By Lemma 3.8 we have the limit diagram

$$
\begin{gathered}
M \otimes \mathcal{W}_{D(2)} \frac{\operatorname{id}_{M} \otimes \mathcal{W}_{\left(d_{1}, d_{2}\right) \in D^{2} \mapsto\left(d_{1}, d_{1} d_{2}\right) \in D(2)}}{M \otimes \mathcal{W}_{D^{2}}} \underset{\operatorname{id}_{M} \otimes \mathcal{W}_{d \in D \mapsto(0,0) \in D^{2}}}{\stackrel{\operatorname{id}_{M} \otimes \mathcal{W}_{d \in D \mapsto(0, d) \in D^{2}}}{\longrightarrow}} M \otimes \mathcal{W}_{D} .
\end{gathered}
$$

Therefore, we have

$$
M \otimes \mathcal{W}_{D(2)}=\overline{\mathbf{T}}_{M}^{\mathcal{W}_{D}}\left(\tau_{\mathcal{W}_{D}}(M)\right)
$$

while we have

$$
M \otimes \mathcal{W}_{D(2)}=\left(M \otimes \mathcal{W}_{D}\right) \times_{M}\left(M \otimes \mathcal{W}_{D}\right)
$$

Therefore, the desired conclusion follows.

\section{Differential FORMS}

Let $\mathbb{E}$ be a Euclidean $\mathbb{R}$-module which is microlinear and Weil exponentiable.

Definition 4.1 (Differential forms with values in $\mathbb{E}$ ). We denote by $\Omega^{n}(M ; \mathbb{E})$ the intersection of all the following equalizers: 
(1) the equalizer of the exponential transpose

$$
\mathbb{E}^{M \otimes \mathcal{W}_{D^{n}}} \rightarrow \mathbb{E}^{\mathbb{R} \times\left(M \otimes \mathcal{W}_{D^{n}}\right)}
$$

of the composition of

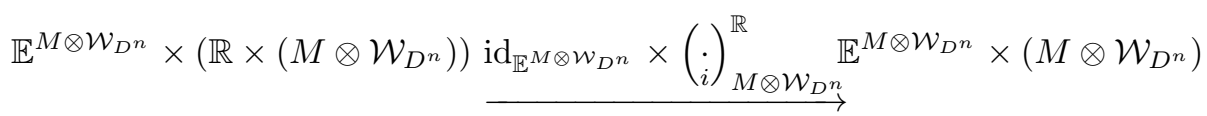

and

$$
\mathbb{E}^{M \otimes \mathcal{W}_{D^{n}}} \times\left(M \otimes \mathcal{W}_{D^{n}}\right) \stackrel{\text { ey }}{\mathbb{E}}
$$

in succession and the exponential transpose

$$
\mathbb{E}^{M \otimes \mathcal{W}_{D^{n}}} \rightarrow \mathbb{E}^{\mathbb{R} \times\left(M \otimes \mathcal{W}_{D^{n}}\right)}
$$

of the composition of

$$
\begin{aligned}
& \mathbb{E}^{M \otimes \mathcal{W}_{D^{n}} \times\left(\mathbb{R} \times\left(M \otimes \mathcal{W}_{D^{n}}\right)\right)} \\
= & \mathbb{R} \times\left(\mathbb{E}^{M \otimes \mathcal{W}_{D^{n}}} \times\left(M \otimes \mathcal{W}_{D^{n}}\right)\right) \stackrel{\mathrm{id}_{\mathbb{R}} \times \mathrm{ev}}{\longrightarrow} \times \mathbb{E}
\end{aligned}
$$

and the scalar multiplication

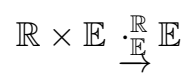

in succession, where $i$ ranges over the natural numbers from 1 to $n$, and the exponential transpose of

$$
(\cdot)_{M \otimes \mathcal{W}_{D^{n}}}^{\mathbb{R}}: \mathbb{R} \times\left(M \otimes \mathcal{W}_{D^{n}}\right) \rightarrow M \otimes \mathcal{W}_{D^{n}}
$$

is

$$
\begin{aligned}
& \alpha_{\mathcal{W}_{\left(a, d_{1}, \ldots, d_{n}\right) \in \mathbb{R} \times D^{n} \rightarrow\left(d_{1}, \ldots, a d_{i}, \ldots, d_{n}\right) \in D^{n}}}(M) \\
& : M \otimes \mathcal{W}_{D^{n}} \rightarrow\left(M \otimes \mathcal{W}_{D^{n}}\right)^{\mathbb{R}}=\left(M \otimes \mathcal{W}_{D^{n}}\right) \otimes \mathcal{W}_{\mathbb{R}} \\
& =M \otimes \mathcal{W}_{D^{n} \times \mathbb{R}}=M \otimes \mathcal{W}_{\mathbb{R} \times D^{n}},
\end{aligned}
$$

(2) the equalizer of the exponential transpose

$$
\mathbb{E}^{M \otimes \mathcal{W}_{D^{n}}} \rightarrow \mathbb{E}^{M \otimes \mathcal{W}_{D^{n}}}
$$

of the composition of

$$
\mathbb{E}^{M \otimes \mathcal{W}_{D^{n}}} \times\left(M \otimes \mathcal{W}_{D^{n}}\right) \underset{\operatorname{id}_{\mathbb{E}^{M \otimes \mathcal{W}_{D^{n}}}} \times\left(\cdot^{\sigma}\right)_{M \otimes \mathcal{W}_{D^{n}}}}{\longrightarrow} \mathbb{E}^{M \otimes \mathcal{W}_{D^{n}}} \times\left(M \otimes \mathcal{W}_{D^{n}}\right)
$$

and

$$
\mathbb{E}^{M \otimes \mathcal{W}_{D^{n}}} \times\left(M \otimes \mathcal{W}_{D^{n}}\right) \stackrel{\text { ey }}{\mathbb{E}}
$$

in succession and the exponential transpose

$$
\mathbb{E}^{M \otimes \mathcal{W}_{D^{n}}} \rightarrow \mathbb{E}^{M \otimes \mathcal{W}_{D^{n}}}
$$

of the composition of

$$
\mathbb{E}^{M \otimes \mathcal{W}_{D^{n}}} \times\left(M \otimes \mathcal{W}_{D^{n}}\right) \stackrel{\text { ey }}{\mathbb{E}}
$$

and

$$
\mathbb{E} \underset{\left(\epsilon_{\sigma}\right)_{\mathbb{E}}}{\longrightarrow} \mathbb{E}
$$


in succession, where $\sigma$ ranges over all the permutations of the set $\{1, \ldots, n\}$, $\epsilon_{\sigma}$ is the signature of $\sigma,\left(\epsilon_{\sigma}\right)_{\mathbb{E}}$ is the scalar multiplication by $\epsilon_{\sigma}$, and

$$
\left(\cdot{ }^{\sigma}\right)_{M \otimes \mathcal{W}_{D^{n}}}: M \otimes \mathcal{W}_{D^{n}} \rightarrow M \otimes \mathcal{W}_{D^{n}}
$$

is

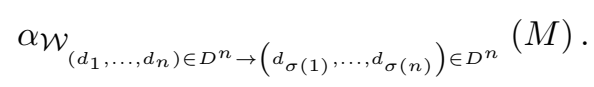

Definition 4.2 (Infinitesimal integration of differential forms). We define a morphism

$$
\int_{M, \mathbb{E}}^{n}:\left(M \otimes \mathcal{W}_{D^{n}}\right) \times \Omega^{n}(M ; \mathbb{E}) \rightarrow \mathbb{E}
$$

in $\mathcal{K}$ to be the composition of

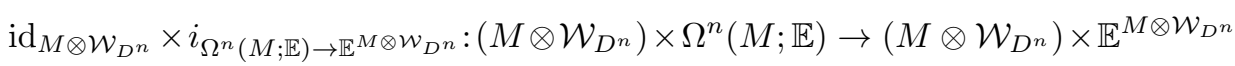
and

$$
\mathrm{ev}:\left(M \otimes \mathcal{W}_{D^{n}}\right) \times \mathbb{E}^{M \otimes \mathcal{W}_{D^{n}}} \rightarrow \mathbb{E}
$$

in succession, where

$$
i_{\Omega^{n}(M ; \mathbb{E}) \rightarrow \mathbb{E}^{M \otimes \mathcal{W}_{D^{n}}}}: \Omega^{n}(M ; \mathbb{E}) \rightarrow \mathbb{E}^{M \otimes \mathcal{W}_{D^{n}}}
$$

is the canonical injection.

Remark 4.3. We should point out that the orthodox definition of the infinitesimal integration of differential forms in synthetic differential geometry, such as seen in Chapter 4 of [2], is unnecessarily decorated with redundant fringes. Therein, it is defined as a mapping

$$
\left(M \otimes \mathcal{W}_{D^{n}}\right) \times \Omega^{n}(M ; \mathbb{E}) \rightarrow \mathbb{E} \otimes \mathcal{W}_{D^{n}},
$$

where the mapping factors through the canonical injection

$$
\operatorname{hom}\left(\mathbb{E} \otimes \mathcal{W}_{D^{n}}\right) \rightarrow \mathbb{E} \otimes \mathcal{W}_{D^{n}}
$$

with hom $\left(\mathbb{E} \otimes \mathcal{W}_{D^{n}}\right)$ being the homogeneous subobject of $\mathbb{E} \otimes \mathcal{W}_{D^{n}}$. Since

$$
\operatorname{hom}\left(\mathbb{E} \otimes \mathcal{W}_{D^{n}}\right)
$$

is canonically isomorphic to $\mathbb{E}$, such an unnecessarily decoration is to be averted.

It is trivial to see that

Proposition 4.4. The morphism

$$
\int_{M, \mathbb{E}}^{n}:\left(M \otimes \mathcal{W}_{D^{n}}\right) \times \Omega^{n}(M ; \mathbb{E}) \rightarrow \mathbb{E}
$$

satisfies the following properties:

(1) the composition of morphisms

$$
\begin{aligned}
& (\cdot)_{M \otimes \mathcal{W}_{D^{n}}}^{\mathbb{R}} \times \operatorname{id}_{\Omega^{n}(M ; \mathbb{E})}: \mathbb{R} \times\left(M \otimes \mathcal{W}_{D^{n}}\right) \times \Omega^{n}(M ; \mathbb{E}) \rightarrow\left(M \otimes \mathcal{W}_{D^{n}}\right) \times \Omega^{n}(M ; \mathbb{E}) \\
& \text { and } \\
& \int_{M, \mathbb{E}}^{n}:\left(M \otimes \mathcal{W}_{D^{n}}\right) \times \Omega^{n}(M ; \mathbb{E}) \rightarrow \mathbb{E}
\end{aligned}
$$


in succession is equal to the composition of morphisms

$$
\operatorname{id}_{\mathbb{R}} \times \int_{M, \mathbb{E}}^{n}: \mathbb{R} \times\left(M \otimes \mathcal{W}_{D^{n}}\right) \times \Omega^{n}(M ; \mathbb{E}) \rightarrow \mathbb{R} \times \mathbb{E}
$$

and

$$
\cdot \mathbb{R}: \mathbb{R} \times \mathbb{E} \rightarrow \mathbb{E}
$$

in succession,

(2) the composition of morphisms

$$
\begin{aligned}
& \left({ }^{\sigma}\right)_{M \otimes \mathcal{W}_{D^{n}}} \times \operatorname{id}_{\Omega^{n}(M ; \mathbb{E})}:\left(M \otimes \mathcal{W}_{D^{n}}\right) \times \Omega^{n}(M ; \mathbb{E}) \rightarrow\left(M \otimes \mathcal{W}_{D^{n}}\right) \times \Omega^{n}(M ; \mathbb{E}) \\
& \text { and } \\
& \qquad \int_{M, \mathbb{E}}^{n}:\left(M \otimes \mathcal{W}_{D^{n}}\right) \times \Omega^{n}(M ; \mathbb{E}) \rightarrow \mathbb{E}
\end{aligned}
$$

in succession is equal to the composition of morphisms

$$
\int_{M, \mathbb{E}}^{n}:\left(M \otimes \mathcal{W}_{D^{n}}\right) \times \Omega^{n}(M ; \mathbb{E}) \rightarrow \mathbb{E}
$$

and

$$
\stackrel{\mathbb{E}\left(\epsilon_{\sigma}\right)_{\mathbb{E} \otimes \mathcal{W}_{D^{n}}}^{\longrightarrow}}{\longrightarrow}
$$

in succession.

As could have been expected, we have

Theorem 4.5 (The fundamental theorem on differential forms). Given an $o b$ ject $X$ in $\mathcal{K}$ and a morphism

$$
\varphi:\left(M \otimes \mathcal{W}_{D^{n}}\right) \times X \rightarrow \mathbb{E} \otimes \mathcal{W}_{D^{n}}
$$

if the morphism (4.1) satisfies the two conditions in Proposition 4.4 with $\Omega^{n}(M ; \mathbb{E})$ replaced by $X$ and $\int_{M, \mathbb{E}}^{n}$ replaced by $\varphi$, then there exists a unique morphism

$$
\widehat{\varphi}: X \rightarrow \Omega^{n}(M ; \mathbb{E})
$$

such that $\varphi$ is equal to the composition of morphisms

$$
\operatorname{id}_{M \otimes \mathcal{W}_{D^{n}}} \times \widehat{\varphi}:\left(M \otimes \mathcal{W}_{D^{n}}\right) \times X \rightarrow\left(M \otimes \mathcal{W}_{D^{n}}\right) \times \Omega^{n}(M ; \mathbb{E})
$$

and

$$
\int_{M, \mathbb{E}}^{n}:\left(M \otimes \mathcal{W}_{D^{n}}\right) \times \Omega^{n}(M ; \mathbb{E}) \rightarrow \mathbb{E}
$$

in succession.

Proof. The theorem follows rather directly from the universal construction of $\Omega^{n}(M ; \mathbb{E})$. Take the exponential transpose

$$
\widetilde{\varphi}: X \rightarrow\left(\mathbb{E} \otimes W_{D^{n}}\right)^{M \otimes W_{D^{n}}}
$$

of (4.1), which factors, by the two conditions on $\varphi$, into a morphism

$$
\widehat{\varphi}: X \rightarrow \Omega^{n}(M ; \mathbb{E})
$$

followed by the canonical monomorphism

$$
\Omega^{n}(M ; \mathbb{E}) \rightarrow \mathbb{E}^{M \otimes W_{D^{n}}} .
$$


It is not difficult to see that the above $\widehat{\varphi}$ is the desired unique morphism in the theorem. The details can safely be left to the reader.

\section{THE EXTERIOR DIFFERENTIATION}

Definition 5.1. Given natural numbers $n, i$ with $1 \leq i \leq n+1$, we define a morphism

in $\mathcal{K}$ to be

$$
\left(\int_{M, \mathbb{E}}^{n}\right)_{i}:\left(M \otimes \mathcal{W}_{D^{n+1}}\right) \times \Omega^{n}(M ; \mathbb{E}) \rightarrow \mathbb{E}
$$

$$
\begin{aligned}
& \left(M \otimes \mathcal{W}_{D^{n+1}}\right) \times \Omega^{n}(M ; \mathbb{E}) \\
& \frac{\left(\mathrm{id}_{M} \otimes \mathcal{W}_{\left(d_{1}, \ldots, d_{n+1}\right) \in D^{n+1} \mapsto\left(d_{1}, \ldots, d_{i-1}, d_{i+1}, \ldots, d_{n+1}, d_{i}\right) \in D^{n+1}}\right) \times \mathrm{id}_{\Omega^{n}(M ; \mathbb{E})}}{\left(M \otimes \mathcal{W}_{D^{n+1}}\right) \times \Omega^{n}(M ; \mathbb{E})} \\
= & \left(M \otimes \mathcal{W}_{D^{n+1}}\right) \times\left(\Omega^{n}(M ; \mathbb{E}) \otimes k\right) \\
& \frac{\mathrm{id}_{M \otimes \mathcal{W}_{D^{n+1}}} \times\left(\operatorname{id}_{\Omega^{n}(M ; \mathbb{E})} \otimes\left(k \rightarrow k[X] /\left(X^{2}\right)=\mathcal{W}_{D}\right)\right)}{\longrightarrow} \\
& \left(M \otimes \mathcal{W}_{D^{n+1}}\right) \times\left(\Omega^{n}(M ; \mathbb{E}) \otimes \mathcal{W}_{D}\right) \\
= & \left(\left(M \otimes \mathcal{W}_{D^{n}}\right) \otimes \mathcal{W}_{D}\right) \times\left(\Omega^{n}(M ; \mathbb{E}) \otimes \mathcal{W}_{D}\right) \\
& \left.\left.\int_{M, \mathbb{E}}^{n} \otimes \mathcal{W}_{D^{n}}\right) \times \Omega^{n}(M ; \mathbb{E})\right) \otimes \mathcal{W}_{D} \mathbb{E} \otimes \mathcal{W}_{D} \\
= & \mathbb{E} \otimes \mathbb{E} \rightarrow \mathbb{E},
\end{aligned}
$$

where the last morphism

$$
\mathbb{E} \otimes \mathbb{E} \rightarrow \mathbb{E}
$$

is the second projection, and

$$
k \rightarrow k[X] /\left(X^{2}\right)=\mathcal{W}_{D}
$$

is the canonical morphism.

Notation 5.2. We denote by

$$
\left(\partial_{i}^{n+1}\right)_{M \otimes \mathcal{W}_{D^{n+1}}}
$$

the morphism

$$
\operatorname{id}_{M} \otimes \mathcal{W}_{\left(d_{1}, \ldots, d_{n+1}\right) \in D^{n+1} \mapsto\left(d_{1}, \ldots, d_{i-1}, d_{i+1}, \ldots, d_{n+1}, d_{i}\right) \in D^{n+1}} .
$$

In order to establish the fundamental theorem on exterior differentiation, we need the following two lemmas:

Lemma 5.3. The composition of morphisms

$$
(j)_{M \otimes \mathcal{W}_{D^{n}}}^{\mathbb{R}} \times \operatorname{id}_{\Omega^{n}(M ; \mathbb{E})}: \mathbb{R} \times\left(M \otimes \mathcal{W}_{D^{n+1}}\right) \times \Omega^{n}(M ; \mathbb{E}) \rightarrow\left(M \otimes \mathcal{W}_{D^{n+1}}\right) \times \Omega^{n}(M ; \mathbb{E})
$$

and

$$
\left(\int_{M, \mathbb{E}}^{n}\right)_{i}:\left(M \otimes \mathcal{W}_{D^{n+1}}\right) \times \Omega^{n}(M ; \mathbb{E}) \rightarrow \mathbb{E}
$$


in succession is equal to the composition of morphisms

$$
\operatorname{id}_{\mathbb{R}} \times\left(\int_{M, \mathbb{E}}^{n}\right)_{i}: \mathbb{R} \times\left(M \otimes \mathcal{W}_{D^{n+1}}\right) \times \Omega^{n}(M ; \mathbb{E}) \rightarrow \mathbb{R} \times \mathbb{E}
$$

and

$$
\cdot \mathbb{R}: \mathbb{R} \times \mathbb{E} \rightarrow \mathbb{E}
$$

in succession.

Proof. For $j<i$, it is easy to see that

$$
\begin{aligned}
& \left(\partial_{i}^{n+1}\right)_{M \otimes \mathcal{W}_{D^{n+1}}} \circ(j)_{M \otimes \mathcal{W}_{D^{n+1}}}^{\mathbb{R}} \\
= & \left(\left(\dot{j}_{j}\right)_{M \otimes \mathcal{W}_{D^{n}}}^{\mathbb{R}} \otimes \operatorname{id}_{W_{D}}\right) \circ\left(\operatorname{id}_{\mathbb{R}} \times\left(\partial_{i}^{n+1}\right)_{M \otimes \mathcal{W}_{D^{n+1}}}\right),
\end{aligned}
$$

while, for $j>i$, it is also easy to see that

$$
\begin{aligned}
& \left(\partial_{i}^{n+1}\right)_{M \otimes \mathcal{W}_{D^{n+1}}} \circ(\cdot)_{M \otimes \mathcal{W}_{D^{n+1}}}^{\mathbb{R}} \\
= & \left(\left(\begin{array}{c}
\cdot \\
j-1
\end{array}\right)_{M \otimes \mathcal{W}_{D^{n}}}^{\mathbb{R}} \otimes \operatorname{id}_{W_{D}}\right) \circ\left(\operatorname{id}_{\mathbb{R}} \times\left(\partial_{i}^{n+1}\right)_{M \otimes \mathcal{W}_{D^{n+1}}}\right) .
\end{aligned}
$$

Therefore, for $j \neq i$,

$$
\begin{aligned}
& \left(\int_{M, \mathbb{E}}^{n}\right)_{i} \circ\left((j)_{M \otimes \mathcal{W}_{D^{n+1}}}^{\mathbb{R}} \times \operatorname{id}_{\Omega^{n}(M ; \mathbb{E})}\right) \\
= & (\cdot \mathbb{R}) \circ\left(\operatorname{id}_{\mathbb{R}} \times\left(\int_{M, \mathbb{E}}^{n}\right)_{i}\right)
\end{aligned}
$$

follows directly. It remains to be shown that

$$
\begin{aligned}
& \left(\int_{M, \mathbb{E}}^{n}\right)_{i} \circ\left((\cdot)_{M \otimes \mathcal{W}_{D^{n+1}}}^{\mathbb{R}} \times \operatorname{id}_{\Omega^{n}(M ; \mathbb{E})}\right) \\
= & (\cdot \mathbb{R}) \circ\left(\operatorname{id}_{\mathbb{E}} \times\left(\int_{M, \mathbb{E}}^{n}\right)_{i}\right),
\end{aligned}
$$

which follows readily from

$$
\begin{aligned}
& \left(\partial_{i}^{n+1}\right)_{M \otimes \mathcal{W}_{D^{n+1}}} \circ(\cdot)_{i}^{\mathbb{R}}{ }_{M \otimes \mathcal{W}_{D^{n+1}}}
\end{aligned}
$$

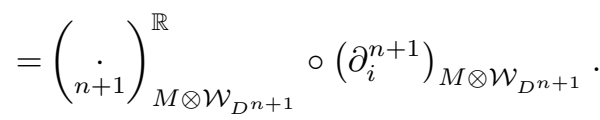


Lemma 5.4. Given a permutation $\sigma$ of $\{1, \ldots, n+1\}$, we have

$$
\begin{aligned}
& \left(\sum_{i=1}^{n+1}(-1)^{i+1}\left(\int_{M, \mathbb{E}}^{n}\right)_{i}\right) \circ\left(\left({ }^{\sigma}\right)_{M \otimes \mathcal{W}_{D^{n+1}}} \times \operatorname{id}_{\Omega^{n}(M ; \mathbb{E})}\right) \\
= & \varepsilon_{\sigma} \sum_{i=1}^{n+1}(-1)^{i+1}\left(\int_{M, \mathbb{E}}^{n}\right)_{i} .
\end{aligned}
$$

Proof. We notice that

$$
\begin{aligned}
& \left(\partial_{i}^{n+1}\right)_{M \otimes \mathcal{W}_{D^{n+1}}} \circ\left(\cdot{ }^{\sigma}\right)_{M \otimes \mathcal{W}_{D^{n+1}}} \\
= & \left(\operatorname{id}_{M} \otimes W_{\left(\delta_{i}^{\sigma}\right)_{D^{n}}}\right)\left(.^{\sigma}\right)_{M \otimes \mathcal{W}_{D^{n+1}}} \circ\left(\partial_{\sigma^{-1}(i)}^{n+1}\right)_{M \otimes \mathcal{W}_{D^{n+1}}},
\end{aligned}
$$

where $\delta_{i}^{\sigma}$ is the permutation of $\{1, \ldots, n\}$ with

$$
\begin{aligned}
& \delta_{i}^{\sigma}(j)=\sigma(j) \text { in case of } j<\sigma^{-1}(i) \text { and } \sigma(j)<i ; \\
& \delta_{i}^{\sigma}(j)=\sigma(j+1) \text { in case of } j \geqq \sigma^{-1}(i) \text { and } \sigma(j)<i ; \\
& \delta_{i}^{\sigma}(j)=\sigma(j)-1 \text { in case of } j<\sigma^{-1}(i) \text { and } \sigma(j) \geqq i ; \\
& \delta_{i}^{\sigma}(j)=\sigma(j+1)-1 \text { in case of } j \geqq \sigma^{-1}(i) \text { and } \sigma(j) \geqq i .
\end{aligned}
$$

We notice also that

$$
\begin{aligned}
& \left(\int_{M, \mathbb{E}}^{n}\right) \circ\left(\left(\delta_{i}^{\sigma}\right)_{M \otimes \mathcal{W}_{D^{n}}} \times \operatorname{id}_{\Omega^{n}(M ; \mathbb{E})}\right) \\
= & \varepsilon_{\delta_{i}^{\sigma}} \int_{M, \mathbb{E}}^{n}
\end{aligned}
$$

and

$$
\varepsilon_{\delta_{i}^{\sigma}}=(-1)^{\sigma^{-1}(i)-i} \varepsilon_{\sigma}
$$

Therefore the desired statement follows.

Theorem 5.5 (The fundamental theorem on exterior differentiation). There exists a unique morphism

$$
\mathbf{d}_{n}: \Omega^{n}(M ; \mathbb{E}) \rightarrow \Omega^{n+1}(M ; \mathbb{E})
$$

in $\mathcal{K}$ such that the the composition of morphisms

$$
\operatorname{id}_{M \otimes \mathcal{W}_{D^{n+1}}} \times \mathbf{d}_{n}:\left(M \otimes \mathcal{W}_{D^{n+1}}\right) \times \Omega^{n}(M ; \mathbb{E}) \rightarrow\left(M \otimes \mathcal{W}_{D^{n+1}}\right) \times \Omega^{n+1}(M ; \mathbb{E})
$$

and

$$
\int_{M, \mathbb{E}}^{n+1}:\left(M \otimes \mathcal{W}_{D^{n+1}}\right) \times \Omega^{n+1}(M ; \mathbb{E}) \rightarrow \mathbb{E}
$$

is equal to the morphism

$$
\sum_{i=1}^{n+1}(-1)^{i+1}\left(\int_{M, \mathbb{E}}^{n}\right)_{i}:\left(M \otimes \mathcal{W}_{D^{n+1}}\right) \times \Omega^{n}(M ; \mathbb{E}) \rightarrow \mathbb{E} .
$$

Proof. This follows easily from Lemmas 5.3 and 5.4 and Theorem 4.5. 


\section{REFERENCES}

[1] A. Kock, Synthetic Differential Geometry, 2nd ed., Cambridge University Press, Cambridge, 2006.

[2] R. Lavendhomme, Basic Concepts of Synthetic Differential Geometry, Kluwer Academic Publishers, Amsterdam, 1996.

[3] H. Nishimura, Differential geometry of microlinear Frölicher spaces II, Int. J. Pure Appl. Math. 64 (2010), 85-102.

[4] H. Nishimura, Axiomatic differential geometry I-1 - towards model categories of differential geometry, Math. Appl. 1 (2012), 171-182.

[5] H. Nishimura, Axiomatic differential geometry II-1 - vector fields, Math. Appl. 1 (2012), 183-195.

Hirokazu Nishimura, Institute of Mathematics, University of Tsukuba, Tsukuba, Ibaraki, 305-8571, Japan

e-mail: logic@math.tsukuba.ac.jp 